\title{
MONOPOLES ON ASYMPTOTICALLY EUCLIDEAN 3-MANIFOLDS
}

\author{
ANDREAS FLOER
}

\begin{abstract}
We consider a generalization of Yang-Mills-Higgs theory on Euclidean $\mathbf{R}^{3}$ to connected sums of $\mathbf{R}^{3}$ with compact closed 3-manifolds.
\end{abstract}

In this note, we describe progress in Yang-Mills-Higgs theory on 3-dimensional Riemannian manifolds. In particular, we are interested in the set of minima of the Yang-Mills action

$$
\mathfrak{A}(A, \Phi)=\int_{M}\left(\left|F_{A}(x)\right|^{2}+\left|\nabla_{A} \Phi(x)\right|^{2}\right) d \mu(x),
$$

where the "Higgs field" $\Phi$ is a section of a metric vector bundle $E$ over $M$ and $A$ is a linear connection on $E$ preserving the metric. For simplicity, we will restrict ourselves to the case where $E=\operatorname{ad}(P)$ is the adjoint bundle of an $\mathrm{SU}_{2}$-principal bundle $P$ over $M$.

The functional $\mathfrak{A}$ has been studied in great detail in the case where $M$ is the Euclidean $\mathbf{R}^{3}$, see [8]. We recall here briefly the main results: The length of the Higgs field $\Phi$ of any finite action configuration $c=(A, \Phi)$ obtains in some sense an asymptotic value $m(c)$ at infinity. For each $m>0$, the space of finite action configurations $c$ with $m(c)=m$ decomposes into a family of components indexed by a "topological charge" $k$. On each of these components, the minima of the action functional (1) can be shown to be solutions of the Bogomolny equation

$$
\mathfrak{b}_{ \pm}(c)=\nabla_{A} \Phi \mp{ }^{*} F_{A}=0
$$

with the sign equal to the sign of $k$. Since reversing the sign of $\Phi$ changes the sign of $k$ while leaving $\mathfrak{A}$ invariant, we can restrict ourselves to the case $k>0$ and write $\mathfrak{b}=\mathfrak{b}_{+}$. The set of gauge equivalence classes of solutions of (2), also called monopoles, is a smooth manifold $\mathcal{M}_{k}$ of dimension $4 k$. It can be described by means of algebraic geometry, see [ 7 and 3]. One usually considers the $(4 k-1)$-dimensional submanifolds $\mathcal{M}_{k}^{m}$ of monopoles [c] with fixed "mass" $m(c)=m$. In fact, one can without loss of generality set $m=1$, since a scaling involving a dilation of $\mathbf{R}^{3}$ shows that $\mathcal{M}_{k}^{m} \cong \mathcal{M}_{k}^{1}$ for all $m>0$.

In order to generalize these ideas, we replace $\mathbf{R}^{3}$ by an asymptotically flat manifold $M$, see [9]. This means that $M$ is the connected sum of $\mathbf{R}^{3}$ with a compact manifold, equipped with a metric which at the end of $M$ is a perturbation of the Euclidean metric in a certain sense. Since in this

Received by the editors July 28, 1986.

1980 Mathematics Subject Classification (1985 Revision). Primary 53C05; Secondary 57M99. 
case, the methods of algebraic geometry do not apply any more, we pursue an entirely analytic approach. At first sight, an obstruction to applying standard methods of infinite-dimensional topology to this problem is the fact that due to the lack of decay conditions for finite action configurations $(A, \Phi)$ at the ends, the function $\mathfrak{b}$ is not defined on a Banach space. However, it turns out that this difficulty is entirely due to the action of the gauge group. As we prove in [4], the space $\mathcal{Q}_{k}$ of gauge equivalence classes of finite action configuration carries a natural Hilbert manifold structure, so that the gauge equivariant function $\mathfrak{b}$ yields a smooth Fredholm section of a smooth bundle over $\mathcal{Q}$. Its index can be determined to be $4 k$.

Since $m(c)$ is gauge invariant, $\mathcal{Q}$ foliates into 1-codimensional submanifolds $\mathcal{Q}^{m}$. Our main result is that certain parts of the zero set $\mathcal{M}_{k}^{m}$ of $\mathfrak{b}$ on $\mathcal{Q}_{k}^{m}$ are regular, i.e., they are locally $(4 k-1)$-dimensional smooth manifolds, and can be described in terms of the space of monopoles on Euclidean $\mathbf{R}^{3}$ and the topology of $M$. In particular, the homology of $M$ enters at this point. For simplicity, we restrict ourselves here to the case $k=1$ (see [5] for general charges). We can then define a real number $R([c])$ measuring the "distance" of the monopole from the center of $M$.

THEOREM. If $M$ is an asymptotically flat manifold with $H_{1}(M, Z)=0$, then the set $\mathcal{M}^{m}=\mathcal{M}_{1}^{m}$ of self-dual gauge equivalence classes in $\mathcal{Q}^{m}=\mathcal{Q}_{1}^{m}$ is a smooth 3-dimensional manifold if either $m(c)$ or $R(c)$ are large enough. In particular, if $m(c)$ is large enough, we have a diffeomorphism

$$
z: \mathcal{M}^{m} \cong M
$$

and for any mass $m>0$ and $r$ large enough a diffeomorphism

$$
z:\left\{[c] \in \mathcal{M}^{m} \mid R(c)>r\right\} \cong \mathbf{R}^{3}-B_{r} .
$$

In both cases, $z$ is defined by the unique zero of the Higgs field. Moreover, for $m$ small enough, $\mathcal{M}^{m}$ contains a component diffeomorphic to $\mathbf{R}^{3}$ and a compact component $K$.

The diffeomorphism (4) is constructed by a grafting procedure similar to the one used in [8] to construct multimonopole solutions on $\mathbf{R}^{3}$. We define approximately self-dual configurations on $M$ from self-dual configurations on $\mathbf{R}^{3}$ by means of cutoff functions. These are then deformed into self-dual configurations using a variant of the implicit function theorem. The same method is used in the small mass limit. The diffeomorphism (3) is obtained by a limit argument using a scale invariance of the self-duality equation.

Note that by transversality theory, a generic perturbation of the Fredholm section $\mathfrak{b}$ yields a cobordism of $M$ to $\mathbf{R}^{3} \cup K$, which is product outside a compact set. The compact manifolds $K$ are related to flat $\mathrm{SU}_{2}$-connections on $M$, whose relation to the topology of homology 3-spheres has been recently investigated by Casson [1].

One can prove similar statements about the monopole spaces for higher charges. For example, each $\mathcal{M}_{k}^{m}$ contains for $m$ small enough a component diffeomorphic to $\mathcal{M}_{k}^{m}\left(\mathbf{R}^{3}\right)$. Moreover, if $H_{1}(M, \mathbf{Z})$ is nonzero but finite, we can state a result similar to the above theorem with diffeomorphisms replaced 
by coverings with covering group $H_{1}(M, \mathbf{Z})$. If it contains a free part, then the maps in (3) and (4) are not surjective in general; see [5]. If $M$ is any asymptotically flat manifold with nonnegative Ricci curvature, then the Fredholm section $\mathfrak{b}$ is "manifestly" regular on each $\mathcal{M}^{m}$. This yields f.e. an alternative proof of the following result:

THEOREM (SEE [10 AND 6]). Every asymptotically Euclidean 3-manifold with nonnegative Ricci curvature is diffeomorphic to $\mathbf{R}^{3}$.

Finally, we should note that generalizations of Euclidean Yang-Mills-Higgs theory have been pursued in a different direction by $\mathrm{A}$. Chakrabarti [2], replacing $\mathbf{R}^{3}$ by certain hyperbolic manifolds, and using essentially methods of algebraic geometry.

The author wishes to thank C. Taubes for suggesting this problem to him and for his support during the research.

\section{REFERENCES}

1. C. Casson, Lectures given at the MSRI, Berkeley, Spring, 1985.

2. A. Chakrabarti, Construction of hyperbolic monopoles, preprint, CNRS, 1982.

3. S. K. Donaldson, Nahm's equation and the classification of monopoles, Comm. Math. Phys. 96 (1984), 387-407.

4. A. Floer, The configuration space of Yang-Mills-Higgs theory on asymptotically flat manifolds, Comm. Math. Phys. (to appear).

5. , Monopoles on asymptotically flat manifolds, Comm. Math. Phys. (to appear).

6. R. S. Hamilton, Three-manifolds with positive Ricci curvature, J. Differential Geom. 17 (1982), 255-306.

7. N. Hitchin, Monopoles and geodesics, Comm. Math. Phys. 83 (1982), 579-602.

8. A. Jaffe and C. Taubes, Vortices and monopoles, Birkhäuser, Boston, 1980.

9. T. Parker and C. Taubes, On Witten's proof of the positive energy conjecture, Comm. Math. Phys. 84 (1982), 223-238.

10. R. Schoen and S. T. Yau, Complete three dimensional manifolds of positive Ricci curvature and scalar curvature, Seminar on Differential Geometry (S. T. Yau, ed.), Princeton, N. J., 1982.

Department of Mathematics, State University of NeW York at Stony BROOK, STONY BROOK, NEW YORK 11794

Current address: Courant Institute, New York University, 251 Mercer Street, New York, New York 10012 
